\title{
Methanol detection in M 82
}

\author{
S. Martín ${ }^{1}$, J. Martín-Pintado ${ }^{2}$, and R. Mauersberger ${ }^{1}$ \\ 1 Instituto de Radioastronomía Milimétrica, Avda. Divina Pastora 7, Local 20, 18012 Granada, Spain \\ e-mail: martin@iram.es \\ 2 Departamento de Astrofísica Molecular e Infrarroja, Instituto de Estructura de la Materia, CSIC, Serrano 121, 28006 Madrid, Spain
}

Received 14 December 2005 / Accepted 7 March 2006

\section{ABSTRACT}

Context. The nuclear starburst region in M 82 shows systematical low abundances of some complex molecules when compared with other starburst galaxies. This is likely related to a presumably photodissociation dominated environment. In particular, methanol is known to show relatively low abundance because it is easily photodissociated.

Aims. We present a multilevel study of the emission of methanol, detected for the first time in this galaxy, and discuss the origin of its emission. Methods. Observations of three transitions of $\mathrm{CH}_{3} \mathrm{OH}$ towards the center and two positions around the nucleus of $\mathrm{M} 82$ are presented. Two different components are found, one with high excitation $\left(n\left(\mathrm{H}_{2}\right) \sim 10^{6} \mathrm{~cm}^{-3}, T_{\text {rot }} \sim 20 \mathrm{~K}\right)$ and the other with low excitation $\left(n\left(\mathrm{H}_{2}\right) \sim 10^{4} \mathrm{~cm}^{-3}\right.$, $\left.T_{\text {rot }} \sim 5 \mathrm{~K}\right)$.

Results. The high observed methanol abundance of a few $10^{-9}$ can only be explained if injection of methanol from dust grains is taken into account. While the overall $\left[\mathrm{CH}_{3} \mathrm{OH}\right] /\left[\mathrm{NH}_{3}\right]$ ratio is much larger than observed towards other starbursts, the dense high excitation component shows a similar value to that found in NGC 253 and Maffei 2.

Conclusions. Our observations suggest the molecular material in M 82 to be formed by dense warm cores, shielded from the UV radiation and similar to the molecular clouds in other starbursts, surrounded by a less dense photodissociated halo. The dense warm cores are likely the location of recent and future star formation within M 82.

Key words. ISM: molecules - galaxies: abundances - galaxies: individual: M 82 - galaxies: ISM - galaxies: starburst

\section{Introduction}

The nuclear environment of M 82 (NGC 3034, Arp 337, 3C231) houses one of the brightest extragalactic IRAS sources (Soifer et al. 1989) and represents an archetype of a nearby $(D=3.6 \mathrm{Mpc}$, Freedman et al. 1994) nuclear starburst galaxy, where stars are forming at a rate of $\sim 9 M_{\odot} \mathrm{yr}^{-1}$ (Strickland et al. 2004). The large supply of molecular gas within the inner few hundred parsecs has been extensively studied in $\mathrm{CO}$ and other molecular tracers (e.g. Mao et al. 2000). Abundances relative to $\mathrm{H}_{2}$ of some complex molecules like $\mathrm{SiO}, \mathrm{CH}_{3} \mathrm{OH}, \mathrm{HNCO}$, $\mathrm{CH}_{3} \mathrm{CN}$ and $\mathrm{NH}_{3}$ are systematically low in comparison to similar starburst prototypes, e.g. NGC 253 (Takano et al. 2002; Martín et al. 2006). These chemical differences have been interpreted as a consequence of nuclear starburst in $\mathrm{M} 82$ being in a more evolved stage than that in NGC 253 (Wang et al. 2004). Observations of HCO (García-Burillo et al. 2002) suggest that photo-dissociation dominates the heating and the chemistry of the molecular material in the nuclear region of $\mathrm{M} 82$.

Methanol $\left(\mathrm{CH}_{3} \mathrm{OH}\right)$ emission is observed in a wide range of physical conditions within the Galactic and extragalactic interstellar medium (Menten et al. 1988; Henkel et al. 1987; Hüttemeister et al. 1997) and also in comets (Bockelée-Morvan et al. 1991). The observed gas-phase abundances range from a few $\sim 10^{-9}$ in dark clouds (Ohishi et al. 1992) and photo-dissociation regions (PDRs) (Jansen et al. 1995) reaching up to $\sim 10^{-6}$ in hot molecular cores (Sutton et al. 1995; Nummelin et al. 2000) and Galactic Center molecular clouds (Requena-Torres et al. 2006, in preparation). $\mathrm{CH}_{3} \mathrm{OH}$ is, after $\mathrm{H}_{2} \mathrm{O}$, the most abundant known constituent of interstellar ices (Allamandola et al. 1992). Observed solid-state $\mathrm{CH}_{3} \mathrm{OH}$ abundances are 1 to 4 order of magnitude larger than those in gasphase (Ehrenfreund \& Charnley 2000; Schöier et al. 2002). This fact, as well as the difficulties of gas-phase chemical models to produce fractional abundances larger than a few $10^{-9}$ (Lee et al. 1996), supports the assumption that large abundances of methanol are due to its injection into the gas-phase via evaporation and/or disruption of ice mantles (Millar et al. 1991; Charnley et al. 1995).

Since methanol is easily photodissociated by UV radiation as observed in galactic PDRs (Hartquist et al. 1995; Le Teuff et al. 2000), one would expect that M 82 will show very low abundances. In this letter we present first detections of $\mathrm{CH}_{3} \mathrm{OH}$ towards the nuclear region of $\mathrm{M} 82$. The fractional abundance of $\mathrm{CH}_{3} \mathrm{OH}$, detected at three positions, is larger than expected if the molecular composition in the nucleus of M 82 is exclusively dominated by PDRs. This fact indicates the presence of a significant fraction of dense gas well shielded from the UV radiation. 


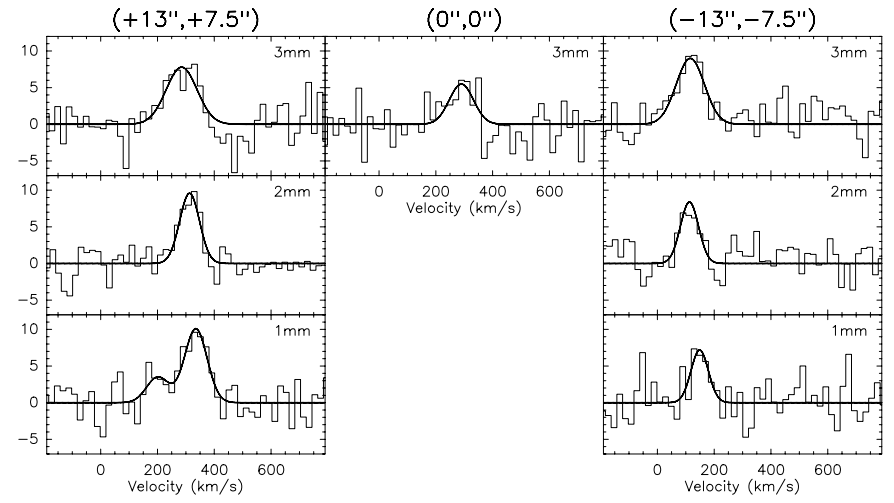

Fig. 1. Observed methanol lines towards three positions in the nucleus of $\mathrm{M}$ 82. The spectra, in $T_{\mathrm{MB}}(\mathrm{mK})$ scale, have been smoothed to a $20 \mathrm{~km} \mathrm{~s}^{-1}$ velocity resolution.

\section{Observations and results}

The observations were carried out in July and December 2004 with the IRAM $30 \mathrm{~m}$ telescope on Pico Veleta, Spain. Three methanol lines at $96.7,157.2$ and $241.7 \mathrm{GHz}$ were observed. The beam sizes of the telescope at these frequencies are $25^{\prime \prime}, 16^{\prime \prime}$ and $10^{\prime \prime}$, respectively. Observations were made in symmetrical wobbler switching mode with a throw of $4^{\prime}$ in azimuth and a frequency of $0.5 \mathrm{~Hz}$. We used the $512 \times$ $1 \mathrm{MHz}$ filter bank for the $3 \mathrm{~mm}$ and two $256 \times 4 \mathrm{MHz}$ filter banks for the 2 and $1 \mathrm{~mm}$ lines. The spectra were calibrated using a dual load system. System temperatures in $T_{\mathrm{MB}}$ were $\sim 200 \mathrm{~K}(3 \mathrm{~mm}), 370 \mathrm{~K}(2 \mathrm{~mm})$ and $630 \mathrm{~K}(1 \mathrm{~mm})$. The nominal pointing position was $\alpha_{J 2000}=09^{\mathrm{h}} 55^{\mathrm{m}} 51^{\mathrm{s}} .9, \delta_{J 2000}=$ $69^{\circ} 40^{\prime} 47^{\prime \prime}$. 1 , corresponding approximately to the $2.2 \mu \mathrm{m}$ emission peak (Dietz et al. 1986; Joy et al. 1987). Continuum cross-scans of point sources made every $2 \mathrm{~h}$ led to a pointing accuracy of $\sim 3^{\prime \prime}$. The central and two offset positions were observed. The North-East (NE at $+13^{\prime \prime},+7.5^{\prime \prime}$ ) and South-West (SW at $-13^{\prime \prime},-7.5^{\prime \prime}$ ) positions correspond approximately to the $\mathrm{HCO}$ emission peaks seen in the interferometric maps by García-Burillo et al. (2002).

Figure 1 shows the observed $\mathrm{CH}_{3} \mathrm{OH}$ line profiles, and Table 1 summarizes the parameters derived from the Gaussian fits to the observed lines in each position. Towards the central position, the emission peaks at $290 \mathrm{~km} \mathrm{~s}^{-1}$ suggesting that most of the emission in the $25^{\prime \prime}$ beam is dominated by the NE clump. Towards the other observed positions the linewidth of the $3 \mathrm{~mm}$ transitions are broader than those of the 2 and $1 \mathrm{~mm}$ lines. This difference is likely due to the larger beam size at $3 \mathrm{~mm}$ detecting a more extended component.

The seemingly low radial velocity of the $3 \mathrm{~mm}$ transition towards the NE position is due to a Gaussian fit to a non-Gaussian profile. In fact, the emission peaks at higher velocities than that of the fit. On the other hand, the systematically higher velocities shown by the $1 \mathrm{~mm}$ transitions at $241.7 \mathrm{GHz}$ are due to the overlap of the different methanol transitions.

To estimate the size of the emitting region, we have followed the procedure used by Mauersberger et al. (2003). From the CS $J=3-2, C^{34} \mathrm{~S} J=2-1$ and $J=3-2$ lines we estimate an equivalent source size for the SW lobe of $\theta_{\mathrm{s}} \sim 12^{\prime \prime}$.
Table 1. Parameters derived from Gaussian fits to the observed lines.

\begin{tabular}{|c|c|c|c|c|c|c|}
\hline $\mathrm{CH}_{3} \mathrm{OH}$ & $\begin{array}{c}\text { Frequency }^{a} \\
\quad(\mathrm{GHz})\end{array}$ & $\underset{\mathrm{mK} \mathrm{km} \mathrm{s}^{-1}}{\int T_{\mathrm{MB}} \mathrm{d} v}$ & $\begin{array}{c}V_{\mathrm{LSR}} \\
\mathrm{km} \mathrm{s}^{-1}\end{array}$ & $\begin{array}{c}\Delta v_{1 / 2} \\
\mathrm{~km} \mathrm{~s}^{-1}\end{array}$ & $\begin{array}{r}T_{\mathrm{MB}} \\
\mathrm{mK}\end{array}$ & $\begin{array}{r}\mathrm{rms}^{b} \\
\mathrm{mK}\end{array}$ \\
\hline & \multicolumn{6}{|c|}{$\left(+13^{\prime \prime},+7.5^{\prime \prime}\right)$} \\
\hline $2_{k}-1_{k}$ & 96.7 & $1070 \pm$ & $285 \pm 11$ & $128 \pm 19$ & 7.8 & 2.5 \\
\hline$J_{0}-J_{-1}$ & 157.3 & $850 \pm 70$ & $313 \pm 4$ & 82 & 9.6 & 1.2 \\
\hline & 241.8 & $1010 \pm 120$ & $335 \pm 6$ & $93 \pm 6$ & 10.1 & 2.2 \\
\hline & 241.9 & $\begin{array}{r}350 \pm 100 \\
\text { Center }\end{array}$ & $\begin{array}{l}310 \pm 10 \\
\left(\mathbf{0}^{\prime \prime}, \mathbf{0}^{\prime \prime}\right)\end{array}$ & 93 & 3.5 & 2.2 \\
\hline $2_{k}-1_{k}$ & 96.7 & $\begin{array}{c}580 \pm 190 \\
\text { SW Lobe }\end{array}$ & $\begin{array}{l}290 \pm 20 \\
\left(-13^{\prime \prime} .-7\right.\end{array}$ & $\begin{array}{l}100 \pm 44 \\
\left.\mathbf{5}^{\prime \prime}\right)\end{array}$ & 5.5 & 2.6 \\
\hline $2_{k}-1_{k}$ & & $1120 \pm 180$ & $116 \pm 8$ & $117 \pm 24$ & 9.0 & 2.5 \\
\hline$J_{0}-J_{-1}$ & 15 & $670 \pm 1$ & $113 \pm$ & $75=$ & 8.4 & 2.0 \\
\hline $5_{k}-4_{k}$ & 241.8 & $550 \pm 120$ & $149 \pm 8$ & $72 \pm 19$ & 7.2 & 2.6 \\
\hline
\end{tabular}

${ }^{a}$ Approximate frequency of each group of transitions, used for velocity scale in Fig. 1.

${ }^{b} \mathrm{rms}$ of the data at a $20 \mathrm{~km} \mathrm{~s}^{-1}$ velocity resolution.

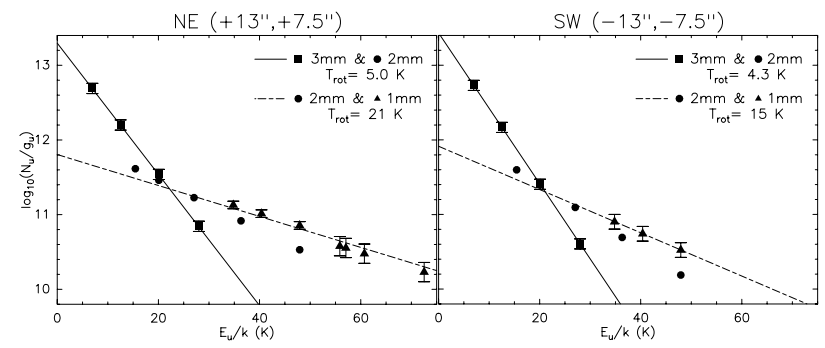

Fig. 2. Rotational diagrams for the the NE and SW positions.

A mean source size of $12^{\prime \prime}$ will be used for all three positions to convert the methanol main beam brightness temperatures in Table 1 into source averaged methanol column densities. This size agrees with the extent of the NE and SW lobes seen in the high resolution HCO maps (García-Burillo et al. 2002).

Figure 2 shows population diagrams for the $\mathrm{NE}$ and SW lobes corrected for beam dilution. Diagrams were plotted by estimating the contribution of each of the transitions to the whole observed profiles (see Martín et al. 2006). Methanol emission has been fitted with a two different temperature components. The fit to the 3 and $2 \mathrm{~mm}$ transitions appear to trace a low excitation gas with a rotational temperature $\left(T_{\text {rot }}\right)$ of 4-5 K and column densities of $N\left[\mathrm{CH}_{3} \mathrm{OH}\right]=3 \times 10^{14} \mathrm{~cm}^{-2}$, similar towards both lobes. Towards the central position, where a $T_{\text {rot }} \sim 4.5 \mathrm{~K}$ has been assumed, we derive a $\mathrm{CH}_{3} \mathrm{OH}$ column density a factor of 2 smaller than towards the other positions. In addition to the low excitation temperature component, the 2 and $1 \mathrm{~mm}$ transitions trace a much higher temperature component with $T_{\text {rot }}=15$ and $21 \mathrm{~K}$, derived for the SW and NE lobes respectively. The NE lobe shows a significantly higher rotational temperature than the SW lobe. Our data clearly show that the methanol emission in M 82 samples two components with very different (low and high) excitation conditions. This indicates the presence of large gradients in the excitation conditions in the molecular gas where methanol emission arises. Assuming a kinetic temperature, $T_{\text {kin }} \sim 30 \mathrm{~K}$ similar to that derived from $\mathrm{NH}_{3}$ observations (Weiß et al. 2001), we find that a large fraction of methanol arises from a relatively low density gas with $n_{\mathrm{H}_{2}} \sim 10^{4} \mathrm{~cm}^{-3}$ and a smaller fraction from denser gas with 
Table 2. Physical parameters derived from observations.

\begin{tabular}{ccccccc}
\hline \hline $\begin{array}{c}\text { Position } \\
\left({ }^{\prime \prime},{ }^{\prime \prime}\right)\end{array}$ & $\begin{array}{c}{ }^{13} \mathrm{CO}_{J=2-1}{ }^{a} \\
\left(\mathrm{~K} \mathrm{~km} \mathrm{~s}^{-1}\right)\end{array}$ & $\begin{array}{c}\left.N 0^{22} \mathrm{~cm}_{2}\right)^{b} \\
N\left[\mathrm{CH}_{3} \mathrm{OH}\right]^{b}\end{array}$ & $\begin{array}{c}T_{\text {rot }} \mathrm{cm}^{-2} \\
(\mathrm{~K})\end{array}$ & $\begin{array}{c}X\left[\mathrm{CH}_{3} \mathrm{OH}\right] \\
\left(10^{-9}\right)\end{array}$ & $\begin{array}{c}n\left(\mathrm{H}_{2}\right)^{c} \\
\left(\mathrm{~cm}^{-3}\right)\end{array}$ \\
\hline$(+13,+7.5)$ & 58.9 & 8.5 & 27 & $5.0 \pm 0.1$ & 3.2 & $5.3-5.4 \times 10^{4}$ \\
$(0,0)$ & 59.8 & 8.6 & $\begin{array}{c}7.5 \\
15\end{array}$ & $\begin{array}{c}21 \pm 1 \\
4.5\end{array}$ & 0.9 & $5.7-1.6 \times 10^{6}$ \\
$(-13,-7.5)$ & 69.0 & 9.9 & 29 & $4.3 \pm 0.1$ & 2.9 & $4.1-4.2 \times 10^{4}$ \\
& & & 5.9 & $15 \pm 1$ & 0.6 & $2.2-1.1 \times 10^{6}$ \\
\hline
\end{tabular}

${ }^{a}$ Integrated intensity from Mao et al. (2000) for a beamwidth of $22^{\prime \prime}$.

${ }^{b}$ Source averaged column density assuming an emission extent of $12^{\prime \prime}$.

${ }^{c}$ Assuming a $T_{\text {kin }}=30-100 \mathrm{~K}$, respectively.

Collisional coefficients from (Pottage et al. 2004)

$n_{\mathrm{H}_{2}}>10^{6} \mathrm{~cm}^{-3}$. The derived $\mathrm{H}_{2}$ densities do not vary significantly if we assume a $T_{\text {kin }} \sim 100 \mathrm{~K}$ as derived for the warmer component observed in CO (Mao et al. 2000).

To estimate the $\mathrm{CH}_{3} \mathrm{OH}$ fractional abundance, we have calculated the column density of molecular hydrogen, $N\left(\mathrm{H}_{2}\right)$, from the ${ }^{13} \mathrm{CO} J=2-1$ observations by Mao et al. (2000) using the conversion $N\left(\mathrm{H}_{2}\right)=3.3 \times 10^{20} \mathrm{~cm}^{-2} \mathrm{I}\left({ }^{13} \mathrm{CO}_{2-1}\right)$ from Mauersberger et al. (2003). Methanol fractional abundances for the low and high excitation components in Table 2 are one order of magnitude higher than the upper abundance limit by Hüttemeister et al. (1997). This disagreement is due to the different $\mathrm{H}_{2}$ column density and the beam filling factor correction applied in this work. The methanol abundance estimate of a few $10^{-9}$ in $M 82$ is still significantly lower than that of $10^{-7.3}-10^{-7.9}$ in other starburst galaxies (Martín et al. 2006).

\section{Discussion}

\subsection{The origin of $\mathrm{CH}_{3} \mathrm{OH}$ in $\mathrm{M}$ 82: photodissociation of core-halo molecular clouds}

The chemical composition of M 82 shows a clear resemblance with that observed in the Orion Bar, prototype of Galactic PDR (Martín et al. 2006), supporting the idea that the chemistry of the nuclear molecular environment of M 82 could be dominated by photo-dissociating radiation (García-Burillo et al. 2002; Mauersberger et al. 2003). This is considered to be the signature of a more evolved stage of its nuclear starburst than in other nearby starburst galaxies such as NGC 253 and NGC 4945 (Wang et al. 2004).

The derived overall fractional abundance of methanol, taking into account the two components (low and high excitation), of $\sim 4 \times 10^{-9}$, similar towards both positions (see Table 2 ), is about a factor 5-10 larger than the values of $0.5-1 \times 10^{-9}$ found in Galactic PDRs like the Orion Bar (Jansen et al. 1995; Johnstone et al. 2003). This suggests that, in addition to photodissociation, another type of chemistry is required to explain the high methanol abundances measured in M 82.

Even the most favorable predictions of gas-phase chemistry models cannot reproduce methanol abundances larger than a few $10^{-9}$ (Lee et al. 1996). Only models which take into account methanol injection from icy mantles into gas-phase are able to produce large scale high abundances (Millar et al. 1991;
Table 3. Methanol to amonia ratio in starbursts.

\begin{tabular}{|c|c|c|c|c|c|}
\hline & \multicolumn{3}{|c|}{ M $82^{a}$} & \multirow[t]{2}{*}{ NGC $253^{b}$} & \multirow[t]{2}{*}{ Maffei 2} \\
\hline & Total & Halo $^{c}$ & Core $^{c}$ & & \\
\hline$N\left[\mathrm{CH}_{3} \mathrm{OH}\right]$ & 34.9 & 29 & 5.9 & 83 & 33 \\
\hline$N\left[\mathrm{NH}_{3}\right]^{d}$ & 36 & $\ll 36$ & $\lesssim 36$ & 440 & 140 \\
\hline$\left[\mathrm{CH}_{3} \mathrm{OH}\right] /\left[\mathrm{NH}_{3}\right]$ & 0.97 & $\gg 0.81$ & $\gtrsim 0.16$ & 0.19 & 0.24 \\
\hline
\end{tabular}

Column densities in units of $10^{13} \mathrm{~cm}^{-2}$.

${ }^{a}$ Data for the SW lobe. ${ }^{b}$ Both lobes included. ${ }^{c}$ Assuming most of the $\mathrm{NH}_{3}$ arises from the high density component. ${ }^{d}$ Data from Weiß et al. (2001); Mauersberger et al. (2003).

Charnley et al. 1995). Thermal evaporation of ices in the nuclear environment of M 82 is unlikely given the low global dust temperature $\left(T_{\mathrm{d}} \sim 50 \mathrm{~K}\right.$, Negishi et al. 2001), which is below the typical critical temperature above which icy mantles evaporation becomes important ( $T_{\text {crit }}>100 \mathrm{~K}$, Isobe 1970; Collings et al. 2004). Like in the nuclei of NGC 253 and the Milky Way, low velocity shocks within molecular cloud complexes seem to be the most likely way of injecting alcohols into the gas-phase (Martín-Pintado et al. 2001; Requena-Torres et al. 2006).

However, the abundance of ammonia, also ejected from icy grain mantles (Flower et al. 1995) and easily photodissociated like methanol (Fuente et al. 1990), is much lower in M 82 than in NGC 253 and Maffei 2. Table 3 shows the comparison of the $\mathrm{CH}_{3} \mathrm{OH}$ and $\mathrm{NH}_{3}$ column densities in $\mathrm{M} 82 \mathrm{SW}$ lobe with those observed in the starburst galaxies NGC 253 and Maffei 2. While the total ratio $\left[\mathrm{CH}_{3} \mathrm{OH}\right] /\left[\mathrm{NH}_{3}\right]$ is similar in NGC 253 and Maffei 2, the overall abundances ratio in M 82 is a factor of 5 larger than in the other galaxies. It is interesting to note that if one considers that all ammonia in $\mathrm{M} 82$ arises from the high density component the $\left[\mathrm{CH}_{3} \mathrm{OH}\right] /\left[\mathrm{NH}_{3}\right]$ abundance ratio is similar to that measured in NGC 253 and Maffei 2.

The emerging scenario from our methanol observations is that the molecular clouds in M 82 have a core-halo structure with a large density contrast. The UV radiation penetrates into the halos, photodissotiating both $\mathrm{NH}_{3}$ and $\mathrm{CH}_{3} \mathrm{OH}$. We find a large methanol to ammonia abundance ratio in the halo as a result of a high temperature chemistry and the slightly different photodissociation cross-sections between both molecules (Le Teuff et al. 2000). On the other hand, in the high density cores, well shielded from the UV radiation, $\mathrm{NH}_{3}$ and $\mathrm{CH}_{3} \mathrm{OH}$ abundances reflect the typical abundance ratio found in other starburst galaxies.

Viti et al. (2002) have followed the complete chemical evolution of a collapsing clump affected by slow shocks and photodissociating UV radiation. In the collapsing phase, molecules which are formed in gas phase freeze out onto the surface of dust grains. Afterwards, slow shocks eject molecules from the grains which are also photodissociated by the UV radiation. Although the conditions in the collapsing core are different than in the molecular clouds in the nucleus of M 82, the model gives insights on the evolution of methanol and ammonia abundances from the shielded cores dominated by the ejection from grains to the low density halo dominated by photochemistry. In their high temperature model (see Fig. 4 from Viti et al. 2002), after the ejection of molecules from grains by shocks, the cores 
show ammonia abundances larger than those of methanol by more than one order the magnitude, similar to what is observed in NGC 253 and the dense warm cores of M 82. As the UV radiation impinges the gas, the $\left[\mathrm{CH}_{3} \mathrm{OH}\right] /\left[\mathrm{NH}_{3}\right]$ ratio increases and after about 100 years the methanol abundance is an order of magnitude larger than that of ammonia. The trend predicted by the model in the abundance ratios is consistent with the idea that the molecular clouds in M 82 are composed of high density cores surrounded by relatively large low density envelopes whose chemistry is dominated by photochemistry. In fact, Mao et al. (2000) also suggested that the bulk of the CO emission from the nuclear region in M 82 stems from a UV-illuminated low density fragmented interclump medium likely associated with the low excitation component observed in methanol.

\subsection{The large scale distribution of methanol in $M 82$}

Recent multiline high-resolution maps towards IC 342 (Meier \& Turner 2005) show a clear difference in the location between the PDRs towards the inner nuclear region and largescale shocks observed along the nuclear arms. The picture of the central parsec of M 82 appears to be fairly different. The emission of methanol seems to follow the double-lobed structure observed in all molecules, which has been interpreted as a molecular ring (Weliachew et al. 1984; Nakai et al. 1987) with a gas depletion in the central region. Observation of molecules claimed to trace PDR show an appreciable emission toward the central region besides the double-peak structure (García-Burillo et al. 2002). Methanol emission in M 82, on the other hand, appears almost fully concentrated toward the lobes at both sides of the nucleus as the profile observed in the central position can be explained from the emission arising mainly from the NE concentration.

Finally, if the methanol emission arising from the densest regions in $\mathrm{M}$ 82, shielded from the $\mathrm{UV}$ radiation, represents the location of recent and/or ongoing star formation, the low column densities measured in this component suggest that M 82 will have less high density gas available for future star formation. This is in contrast with other starburst galaxies like NGC 253 and Maffei 2 and in agreement with M 82 being in a more evolved stage.

Acknowledgements. J.M.P. has been partially supported by the Spanish Ministerio de Educación y Ciencia under projects ESP 200400665, AYA 2002-10113-E and AYA 2003-02785-E.

\section{References}

Allamandola, L. J., Sandford, S. A., Tielens, A. G. G. M., \& Herbst, T. M. 1992, ApJ, 399, 134

Bockelée-Morvan, D., Colom, P., Crovisier, J., Despois, D., \& Paubert, G. 1991, Nature, 350, 318

Charnley, S. B., Kress, M. E., Tielens, A. G. G. M., \& Millar, T. J. 1995, ApJ, 448, 232

Collings, M. P., Anderson, M. A., Chen, R., et al. 2004, MNRAS, 354, 1133

Dietz, R. D., Smith, J., Hackwell, J. A., Gehrz, R. D., \& Grasdalen, G. L. 1986, AJ, 91, 758
Ehrenfreund, P., \& Charnley, S. B. 2000, ARA\&A, 38, 427

Flower, D. R., Pineau des Forêts, G., \& Walmsley, C. M. 1995, A\&A, 294, 815

Freedman, W. L., Hughes, S. M., Madore, B. F., et al. 1994, ApJ, 427, 628

Fuente, A., Martín-Pintado, J., Bachiller, R., \& Cernicharo, J. 1990, A\&A, 237, 471

Fuente, A., García-Burillo, S., Gerin, M., et al. 2005, ApJ, 619, L158

García-Burillo, S., Martín-Pintado, J., Fuente, A., \& Usero, A. 2002, ApJ, 575, L55

Hartquist, T. W., Menten, K. M., Lepp, S., \& Dalgarno, A. 1995, MNRAS, 272, 184

Henkel, C., Jacq, T., Mauersberger, R., Menten, K. M., \& Steppe, H. 1987, A\&A, 188, L1-L4

Hüttemeister, S., Mauersberger, R., \& Henkel, C. 1997, A\&A, 326, 59

Isobe, S. 1970, PASJ, 22, 429

Jansen, D. J., Spaans, M., Hogerheijde, M. R., \& van Dishoeck, E. F. 1995, A\&A, 303, 541

Johnstone, D., Boonman, A. M. S., \& van Dishoeck, E. F. 2003, A\&A, 412, 157

Joy, M., Lester, D. F., \& Harvey, P. M. 1987, ApJ, 319, 314

Lee, H.-H., Bettens, R. P. A., \& Herbst, E. 1996, A\&AS, 119, 111

Le Teuff, Y. H., Millar, T. J., \& Markwick, A. J. 2000, A\&AS, 146, 157

Mao, R. Q., Henkel, C., Schulz, A., et al. 2000, A\&A, 358, 433

Martín, S., Mauersberger, R., Martín-Pintado, J., Henkel, C., \& García-Burillo, S. 2006, ApJS, in press

Martín-Pintado, J., Rizzo, J. R., de Vicente, P., Rodríguez-Fernández, N. J., \& Fuente, A. 2001, ApJ, 548, L65

Mauersberger, R., Henkel, C., Weiß, A., Peck, A. B., \& Hagiwara, Y. 2003, A\&A, 403, 561

Menten, K. M., Walmsley, C. M., Henkel, C., \& Wilson, T. L. 1988, A\&A, 198, 253

Meier, D. S., \& Turner, J. L. 2005, ApJ, 618, 259

Millar, T. J., Herbst, E., \& Charnley, S. B. 1991, ApJ, 369, 147

Nakai, N., Hayashi, M., Handa, T., Sofue, Y., \& Hasegawa, T. 1987, PASJ, 39, 685

Negishi, T., Onaka, T., Chan, K.-W., \& Roellig, T. L. 2001, A\&A, 375, 566

Nummelin, A., Bergman, P., Hjalmarson, Å., et al. 2000, ApJS, 128, 213

Ohishi, M., Irvine, W. M., \& Kaifu, N. 1992, in Astrochemistry of Cosmic Phenomena, ed. P. D. Singh (Dordrecht: Kluwer), IAU Symp., 150, 171

Pottage, J. T., Flower, D. R., \& Davis, S. L. 2004, MNRAS, 352, 39

Requena-Torres, M. A., Martín-Pintado, J., Rodríguez-Franco, A., et al. 2006, submitted

Sandford, S. A., \& Allamandola, L. J. 1993, ApJ, 417, 815

Schöier, F. L., Jørgensen, J. K., van Dishoeck, E. F., \& Blake, G. A. 2002, A\&A, 390, 1001

Soifer, B. T., Boehmer, L., Neugebauer, G., \& Sanders, D. B. 1989, AJ, 98, 766

Strickland, D. K., Heckman, T. M., Colbert, E. J. M., Hoopes, C. G., \& Weaver, K. A. 2004, ApJ, 606, 829

Sutton, E. C., Peng, R., Danchi, W. C., et al. 1995, ApJS, 97, 455

Takano, S., Nakai, N., \& Kawaguchi 2002, PASJ, 54, 195

Viti, S., Natarajan, S., \& Williams, D. A. 2002, MNRAS, 336, 797

Wang, M., Henkel, C., Chin, Y.-N., et al. 2004, A\&A, 422, 883

Weiß, A., Neininger, N., Henkel, C., Stutzki, J., \& Klein, U. 2001, ApJ, 554, L143

Weliachew, L., Fomalont, E. B., \& Greisen, E. W. 1984, A\&A, 137, 335 